\title{
SwissDRG: documentation claire des procédures complexes
}

\author{
Avec l'introduction de SwissDRG, la documentation des mesures médicales diagnos- \\ tiques et thérapeutiques gagne en importance. A terme, seules les prestations docu- \\ mentées seront codées par les spécialistes du codage et remboursées par les répon- \\ dants des coûts. Pour soutenir les médecins dans la documentation des prestations \\ complexes, la FMH met 35 modèles à leur disposition sur son site internet.
}

Petra Ingenpass

Responsable suppléante du domaine SwissDRG de la FMH

Correspondance: Dr Petra Ingenpass Service tarifaire FMH Froburgstrasse 15 CH-4600 Olten Tél. 0313591111 Fax 0313591112 swissdrg@fmh.ch
Depuis le $1^{\text {er }}$ janvier 2011, la classification des procédures CHOP 2011 est publiée dans un volumineux catalogue mis à disposition pour la saisie des prestations hospitalières. Cette version détaillée est le fruit de l'engagement des sociétés de discipline médicale qui, ces dernières années, ont soumis près de 2000 propositions en vue de mieux différencier les procédures existantes ou d'intégrer des traitements qu'il n'était pas possible de reproduire jusque-là. Désormais, un nombre croissant de procédures multimodales et complexes peuvent également être prises en compte. A l'aide de ces codes de diagnostic et de traitement, il est possible de s'assurer qu'à chaque DRG correspond un poids relatif de coût conforme à la prestation. Ainsi, dans le cadre d'une vérification de l'économicité, la documentation médicale sert également à fournir des éléments probants sur un traitement dispensé et éventuellement onéreux.

\section{Le codage des procédures complexes est exigeant}

La saisie des prestations médicales sous DRG pose les nouvelles exigences suivantes: elle doit se faire sans délai et donner la description complète des mesures diagnostiques et thérapeutiques importantes pratiquées lors d'un séjour hospitalier précis. Et elle doit permettre un codage compréhensible sur la base de règlements et de normes de codage applicables.

Dans le cas de mesures diagnostiques et thérapeutiques simples, les spécialistes du codage peuvent facilement identifier celles-ci dans le rapport de sortie ou le dossier médical. Mais pour les traitements complexes, la situation est différente. Les procédures multimodales et complexes se distinguent, et se définissent, par des contenus déterminés ou des caractéristiques minimales. Et ce n'est qu'une fois que certains critères sont réalisés qu'un code peut être attribué.

Ainsi, les codes de procédures interdisciplinaires, complexes ou multimodales, peuvent être définis notamment par les critères et les caractéristiques minimales ci-après:
- le genre / le nombre de spécialités et de domaines thérapeutiques intervenant lors du traitement,

- le genre / le nombre de procédures thérapeutiques mises en œuvre,

- les échelonnements de la durée de traitement ou des unités thérapeutiques.

En bref: il s'agit d'une foule d'informations particulières qui sont présentes à l'esprit de celui ou celle qui effectue le traitement, mais ne se révèlent aux personnes impliquées dans la gestion des données que si les informations sont regroupées sous une forme adéquate. En effet, ce n'est qu'en présence de données compréhensibles qu'il sera possible d'attribuer le code CHOP s'y rapportant. Et seuls les codes documentés de manière transparente et compréhensible n'auront pas à redouter les vérifications des réviseurs du codage ou des assurances. Conclusion: il faut disposer d'une documentation montrant qu'il s'agit bien d'une procédure complexe.

\section{Aides à la documentation pour les procédures complexes}

Afin de pouvoir offrir une aide aux médecins dans la saisie des prestations complexes, la FMH a élaboré des modèles de documentation et les a mis en consultation auprès des sociétés de discipline médicale. Ces modèles comprennent un énoncé clair de toutes les informations relatives au code CHOP concerné; ils sont à la disposition des membres FMH sous www.fmh.ch $\rightarrow$ Tarifs $\rightarrow$ SwissDRG $\rightarrow$ Documentation médicale des codes CHOP complexes. Leur utilisation dans le sens d'une liste de contrôle doit permettre d'éviter que la documentation des procédures complexes ne demande un travail administratif démesuré.

Les modèles de documentation sont des propositions de la FMH et n'ont aucun caractère obligatoire. Des adaptations aux besoins internes des hôpitaux sont possibles, tout comme l'intégration de ces modèles dans un système d'information hospitalier. 